University of North Florida

UNIVERSITY of

NORTH FLORIDA.

UNF Digital Commons

$11-15-2008$

\title{
Relaxations in Ba2BiSb06 Double Complex Perovskite Ceramics
}

Manoel Carvalho Castro Jr.

Carlos William de Araujo Paschoal

Frank C. Snyder

Michael W. Lufaso

University of North Florida, michael.lufaso@unf.edu

Follow this and additional works at: https://digitalcommons.unf.edu/achm_facpub

Part of the Chemistry Commons

\section{Recommended Citation}

Castro, Manoel Carvalho Jr.; Paschoal, Carlos William de Araujo; Snyder, Frank C.; and Lufaso, Michael W., "Relaxations in Ba2BiSb06 Double Complex Perovskite Ceramics" (2008). Chemistry Faculty Publications. 8.

https://digitalcommons.unf.edu/achm_facpub/8

This Article is brought to you for free and open access by the Department of Chemistry at UNF Digital Commons. It has been accepted for inclusion in Chemistry Faculty

Publications by an authorized administrator of UNF Digital Commons. For more information, please contact Digital Projects.

(C) 11-15-2008 All Rights Reserved

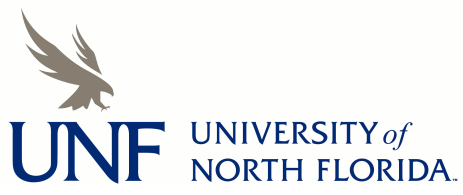




\title{
Relaxations in $\mathrm{Ba}_{2} \mathrm{BiSbO}_{6}$ double complex perovskite ceramics
}

\author{
Manoel Carvalho Castro, Jr., ${ }^{1}$ Carlos William de Araujo Paschoal, ${ }^{1, a)}$ Frank C. Snyder, ${ }^{2}$ \\ and Michael W. Lufaso ${ }^{2}$ \\ ${ }^{1}$ Departamento de Física, Universidade Federal do Maranhão, 65085-580 São Luis-Maranhão, Brazil \\ ${ }^{2}$ Department of Chemistry and Physics, University of North Florida, 1 UNF Drive, Jacksonville, Florida \\ 32224, USA
}

(Received 1 August 2008; accepted 14 October 2008; published online 24 November 2008)

\begin{abstract}
The electric properties of the complex double perovskite $\mathrm{Ba}_{2} \mathrm{BiSbO}_{6}$ have been investigated using impedance spectroscopy in the frequency range from $1 \mathrm{~Hz}$ up to $1 \mathrm{MHz}$ and in the temperature range from room temperature up to $560 \mathrm{~K}$. There are two contributions to the electrical properties due to the grain and grain boundary. The oxygen vacancies play an important role in the conductivity and strongly increase the dielectric constant at high temperatures. The analysis of the frequency dependence of the conductivity clearly shows the structural phase transition of this compound near 515 K. (C) 2008 American Institute of Physics. [DOI: 10.1063/1.3026654]
\end{abstract}

\section{INTRODUCTION}

Perovskite like compounds have found wide use in a variety of technological devices. Several perovskite related compounds have been applied as piezoelectric and pyroelectric transducers, laser hosts, transparent ceramics, electrooptical shutters, second harmonic generators, electrodes, ferroelectrics, resonators, and resistors. ${ }^{1}$ Substitution of the $B$-site cation in perovskite $A B \mathrm{O}_{3}$ compounds by two $B^{\prime}$ and $B^{\prime \prime}$ ions, whose formal oxidation state may be different, has been an important tool for increasing the number of known perovskites and diversity of physical properties, which have increased the number of possible applications. This substitution is generally obtained in 1:1 or 1:2 ratios. In the 1:2 ratio case, the complex perovskites stoichiometry is $A_{3} B^{\prime} B_{2}^{\prime \prime} \mathrm{O}_{9}$ or, conveniently, $A B_{1 / 3}^{\prime} B_{2 / 3}^{\prime \prime} \mathrm{O}_{3}$, with certain compounds exhibiting the extensively studied relaxor effect ${ }^{2-7}$ and dielectric constant and losses that permit applications as microwave dielectrics. ${ }^{8-14}$ For the $1: 1$ ratio case, one common application is as microwave dielectrics. ${ }^{15-19}$ For this ratio the structure normally shows ordering in the $B^{\prime}$ and $B^{\prime \prime}$ ion distributions that occur in an alternate form along with the three crystalline axes. This ordering is commonly called $\mathrm{NaCl}$ ordered and the aristotype structure is cubic with $F m \overline{3} m$ symmetry. Particularly, in the case of complex double perovskite compounds family $\mathrm{Ba}_{2} \mathrm{Bi}^{3+} \mathrm{Me}^{5+} \mathrm{O}_{6}\left(\mathrm{Me}^{5+}=\mathrm{Bi}^{5+}, \mathrm{Sb}^{5+}\right.$, and $\mathrm{Ta}^{5+}$ ), the interest is related to the superconductivity showed by solid solutions involving $\mathrm{BaBiO}_{3}{ }^{20-26}$ Since that was confirmed, the $\mathrm{Bi}^{4+}$ disproportionation ${ }^{27-29}$ in $\mathrm{Bi}^{3+}$ and $\mathrm{Bi}^{5+}$ ions has emerged a very intense interest in related compounds where $\mathrm{Bi}^{5+}$ is substituted for another pentavalent ion such as $\mathrm{Sb}^{5+}$ and $\mathrm{Ta}^{5+}{ }^{30-35}$ This family of compounds shows a rich phase diagram with temperature changes. Each compound shows at least three phases, the aristotype at high temperatures, a rhombohedral $R \overline{3}$ phase at intermediate temperatures, and a monoclinic $I 2 / \mathrm{m}$ phase at low temperatures. ${ }^{30-32}$ $\mathrm{BaBiO}_{3}$ shows these same three phases but the phase transitions occur at high temperatures. At room temperature

${ }^{a)}$ Electronic mail: paschoal@ufma.br.
$\mathrm{BaBiO}_{3}$ is monoclinic with symmetry $I 2 / \mathrm{m}$ and at low temperatures it is monoclinic with $P 2_{1} / n$ symmetry. ${ }^{30}$ The phase transition $R \overline{3} \rightarrow I 2 / m$ is also pressure induced. ${ }^{32,34,35}$ However, investigations on the $\mathrm{Ba}_{2} \mathrm{Bi}^{3+} \mathrm{Me}^{5+} \mathrm{O}_{6}\left(\mathrm{Me}^{5+}=\mathrm{Sb}^{5+}\right.$ and $\mathrm{Ta}^{5+}$ ) ions have focused on the structural properties and structural phase transitions of these compounds. The electrical or vibrational properties have not been extensively investigated and reported to our knowledge. Besides, recent electrical investigations in double complex perovskites in the rf frequency range have showed important features such as defect ordering induced relaxor ferroelectric behavior ${ }^{36}$ and giant dielectric constant. ${ }^{37}$ This work shows for the first time the use of impedance spectroscopy to characterize the temperature dependence of electrical properties of $\mathrm{Ba}_{2} \mathrm{BiSbO}_{6}$ ceramic samples.

\section{EXPERIMENTAL DETAILS}

$\mathrm{Ba}_{2} \mathrm{BiSbO}_{6}$ powder was prepared in air by conventional solid state techniques using appropriate stoichiometric mixtures of high purity $(99.99 \%$, metal basis, Alfa Aesar) $\mathrm{BaCO}_{3}, \mathrm{Bi}_{2} \mathrm{O}_{3}$, and $\mathrm{Sb}_{2} \mathrm{O}_{3}$. The sample was prepared stoichiometrically and ground under acetone with an agate mortar and pestle. The sample was heated for several days in a high form alumina crucible with intermediate regrinding at temperatures up to $850{ }^{\circ} \mathrm{C}$ until the diffraction peaks, established using a Rigaku Ultima III powder x-ray diffractometer, associated with cation ordering no longer changed. A $13 \mathrm{~mm}$ diameter pellet was uniaxially pressed in a steel die using a Carver hydraulic press. The pellet was placed on a bed of sacrificial powder of the same composition to minimize reaction with the alumina crucible and sintered at $850{ }^{\circ} \mathrm{C}$.

Impedance spectroscopy measurements were performed using a Solartron frequency response analyzer model 1260 coupled to a dielectric interface Solartron model 1296. The measurements were carried out in the frequency range from $1 \mathrm{~Hz}$ up to $1 \mathrm{MHz}$ and in the temperature range from room temperature up to $560 \mathrm{~K}$. Silver paste was used to deposit the contacts on the ceramic as parallel electrodes. The tempera- 

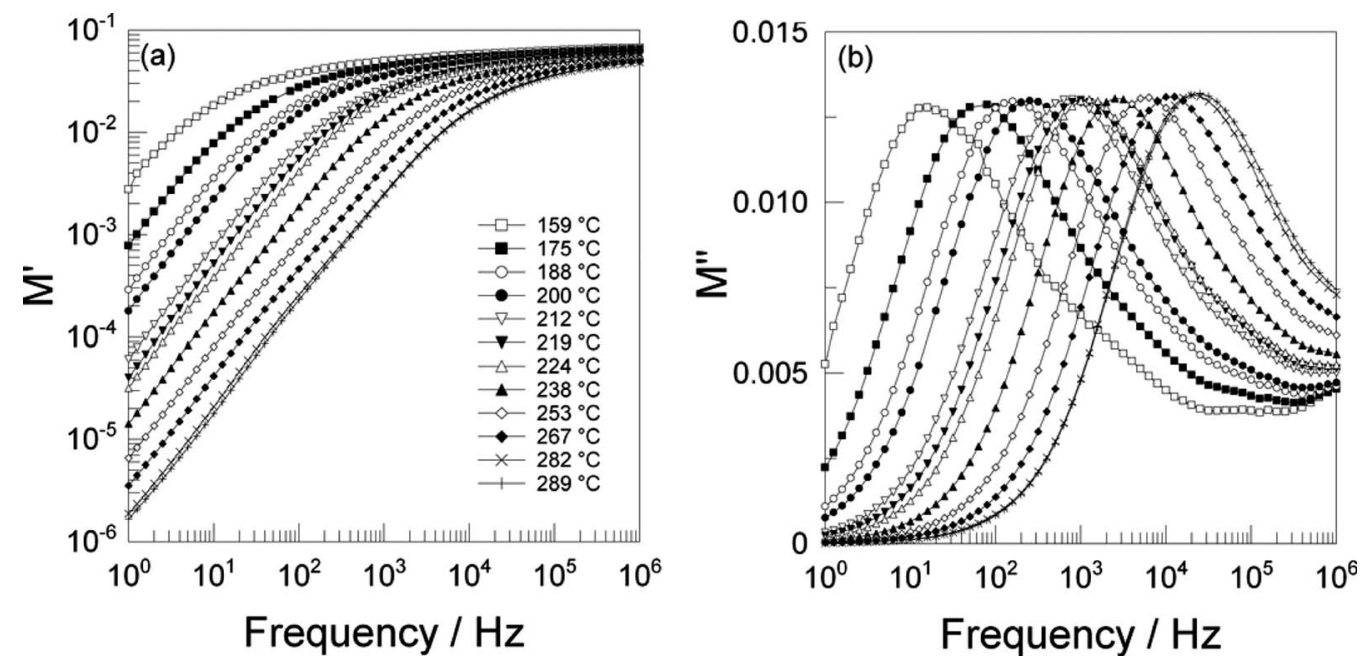

FIG. 1. Frequency dependence of the (a) real part and (b) imaginary part of the electrical modulus of the $\mathrm{Ba}_{2} \mathrm{BiSbO}_{6}$ at several temperature values.

ture measurements were performed in an EDG 10P furnace controlled by proportional-integral-derivative thermal controller. All the measurements were performed under $\mathrm{N}_{2}$ as an inert gas.

\section{RESULTS AND DISCUSSIONS}

Polycrystalline samples may be analyzed by considering the impedance $Z(\omega)$ spectra as an electric modulus $M(\omega)$ spectra in order to emphasize the grain and grain boundary contributions to the electrical properties. ${ }^{38}$ In Fig. 1 we show the electrical modulus response to the $\mathrm{Ba}_{2} \mathrm{BiSbO}_{6}$ as a function of the oscillating frequency of the applied field at several measured temperatures.

In terms of the dielectric constant $\varepsilon(\omega)$, the electric modulus is given by

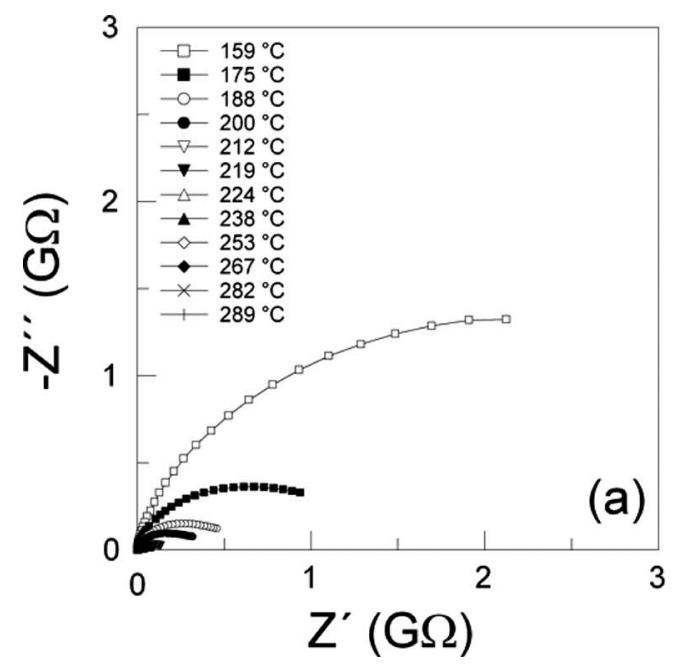

$$
\begin{aligned}
M(\omega)= & M^{\prime}(\omega)+j M^{\prime \prime}(\omega)=\frac{1}{\varepsilon(\omega)} \\
& \rightarrow\left\{\begin{array}{l}
M^{\prime}(\omega)=\frac{\varepsilon^{\prime}(\omega)}{\varepsilon^{\prime}(\omega)^{2}+\varepsilon^{\prime \prime}(\omega)^{2}} \\
M^{\prime \prime}(\omega)=\frac{\varepsilon^{\prime \prime}(\omega)}{\varepsilon^{\prime}(\omega)^{2}+\varepsilon^{\prime \prime}(\omega)^{2}} .
\end{array}\right.
\end{aligned}
$$

Physically, the peak in the imaginary part of the electric modulus defines the regions where the carrier can move at long distances (left to the peak) or confinement (right to the peak). Also, a peak in the $M(\omega)$ imaginary part indicates a dielectric relaxation process in the solid, and the frequency to the maximum indicates the mean relaxation time of this process. As can be seen, the imaginary part of the electric modulus exhibits a very well defined peak. In our case the peak is relatively symmetrical in a considerable frequency range at each measured temperature. This effect is not observed in other double perovskites. ${ }^{39-45}$ As well as in other double perovskites, the frequency at which the maximum

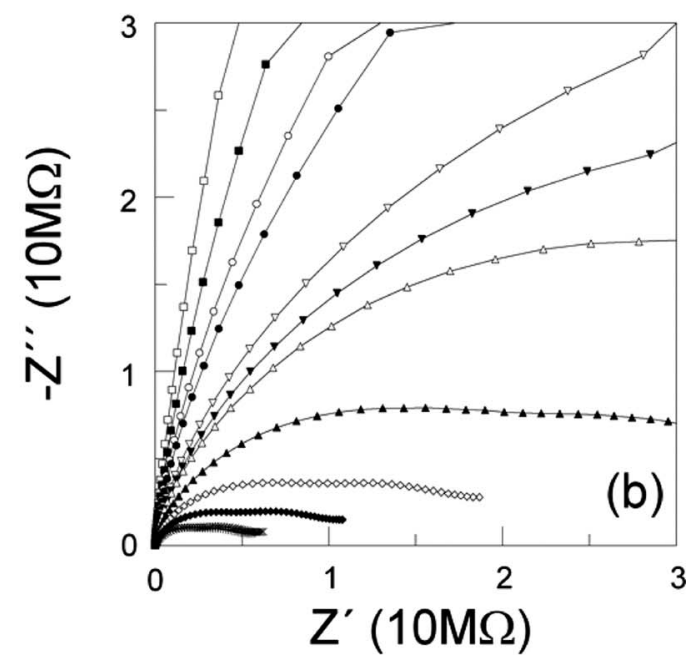

FIG. 2. Nyquist plots of the impedance of the $\mathrm{Ba}_{2} \mathrm{BiSbO}_{6}$ at several temperature values. (a) Plots for all measured temperatures and a (b) zoom for high temperatures. 

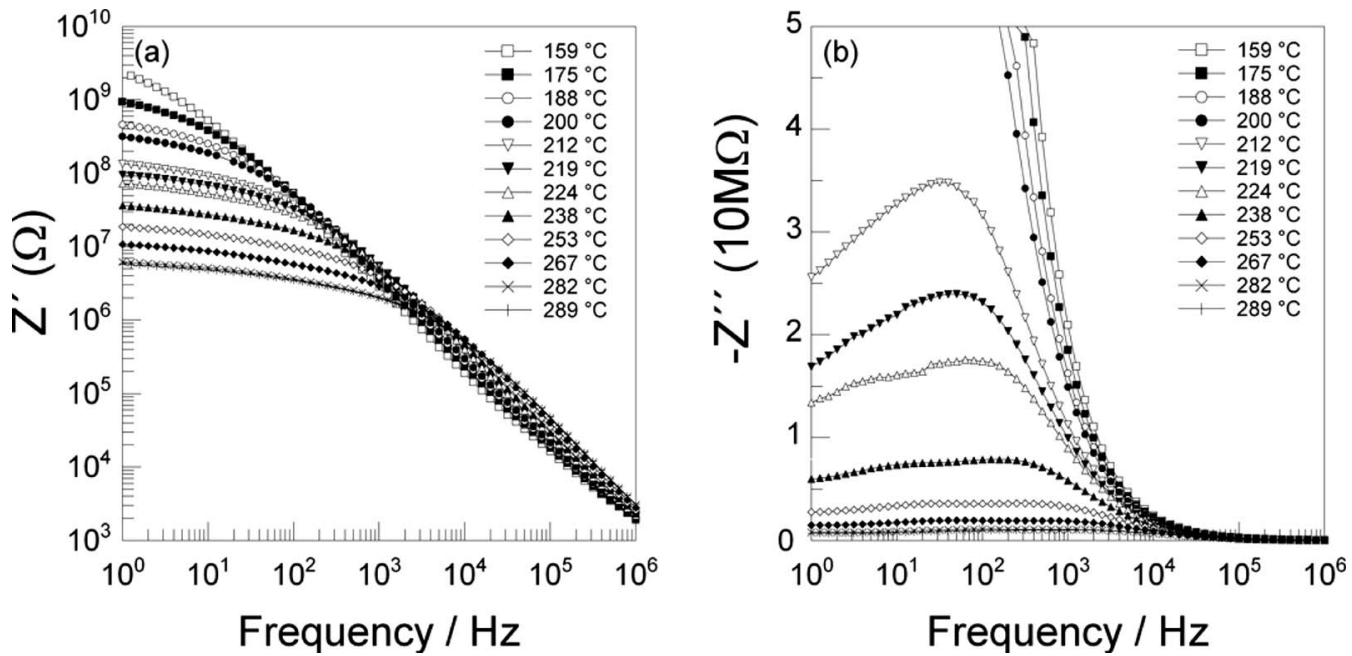

FIG. 3. Bode plots of the impedance of the $\mathrm{Ba}_{2} \mathrm{BiSbO}_{6}$ at several temperature values. (a) Real part of the impedance and (b) a zoom in of the imaginary part of the impedance for high temperatures.

occurs depends on the temperature where the relaxation time is thermally activated. At the right of the main peak a subtle peak whose maxima shift up with the temperature is observed. This indicates a possible second relaxation process, and in order to analyze this we show in Figs. 2 and 3 the Nyquist plot of the impedance and Bode graphs to the real and imaginary parts of the impedance, respectively.

It is clear that there is a new peak in the imaginary part of the impedance [see Fig. 3(b)] evidenced by a new semicircular arc in the Nyquist plot of the impedance [see Fig. 2(b)], implying another relaxation process that is observed when the impedance of the bulk decreases with temperature. These two relaxation processes are due to the bulk (first process) and to the grain boundary (second process). This effect was not observed in other double perovskites, ${ }^{39-45}$ probably due to the high sintering temperature used in these other compounds. In order to analyze the temperature dependence of the relaxation time of these two processes we plot in Fig. 4 the reciprocal temperature dependence of the frequency, where the imaginary parts of the electric modulus and impedance show maxima. Both relaxation processes have a

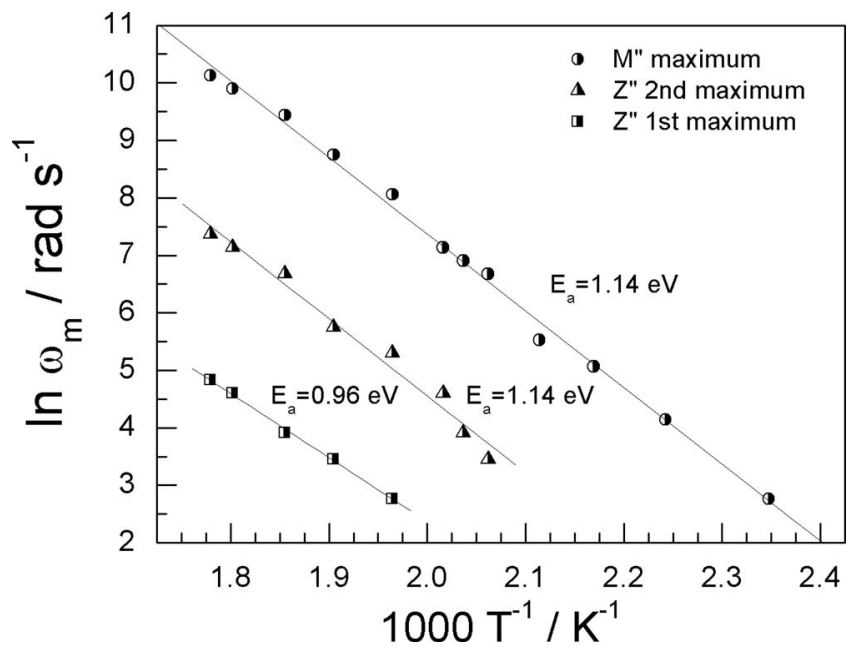

FIG. 4. Arrhenius plots for the frequencies where imaginary parts of the electric modulus and impedance show maxima. mean relaxation time that increases with the temperature exponentially, as suggested by the Arrhenius plot, with activation energies of 1.14 and $0.96 \mathrm{eV}$. The activation energy for the relaxation associated with the bulk is substantially higher than that observed in some other double perovskites, whose $B^{\prime}$ and $B^{\prime \prime}$ ions have the same valence than $\mathrm{Bi}^{3+}$ and $\mathrm{Sb}^{5+}$ such as $\quad \mathrm{Ca}_{2} \mathrm{FeSbO}_{6} \quad(\sim 0.60 \mathrm{eV}),{ }^{43} \quad \mathrm{Ba}_{2} \mathrm{FeSbO}_{6}$ $(\sim 0.75 \mathrm{eV}),{ }^{42}$ and $\mathrm{Ba}_{2} \mathrm{AlNbO}_{6}(\sim 0.49 \mathrm{eV}){ }^{40}$ This bulk activation energy has a magnitude of the order of the oxygen vacancy motion. ${ }^{36}$ The temperature dependence of the dielectric constant is showed in Fig. 5.

The $\mathrm{Ba}_{2} \mathrm{BiSbO}_{6}$ is not ferroelectric ${ }^{46}$ when its crystalline structure is in the nonpolar space group $R \overline{3}$, so we did not expect to observe an intense relaxor peak under temperature changes. However, we can see that the dielectric constant strongly increases at high temperatures. This effect is associated with the conductivity contribution due to the oxygen vacancies.

The conductivity of the $\mathrm{Ba}_{2} \mathrm{BiSbO}_{6}$ was obtained using the Maxwell relation $\sigma(\omega)=j \omega \varepsilon_{o} \varepsilon(\omega)$, where $j$ is the pure

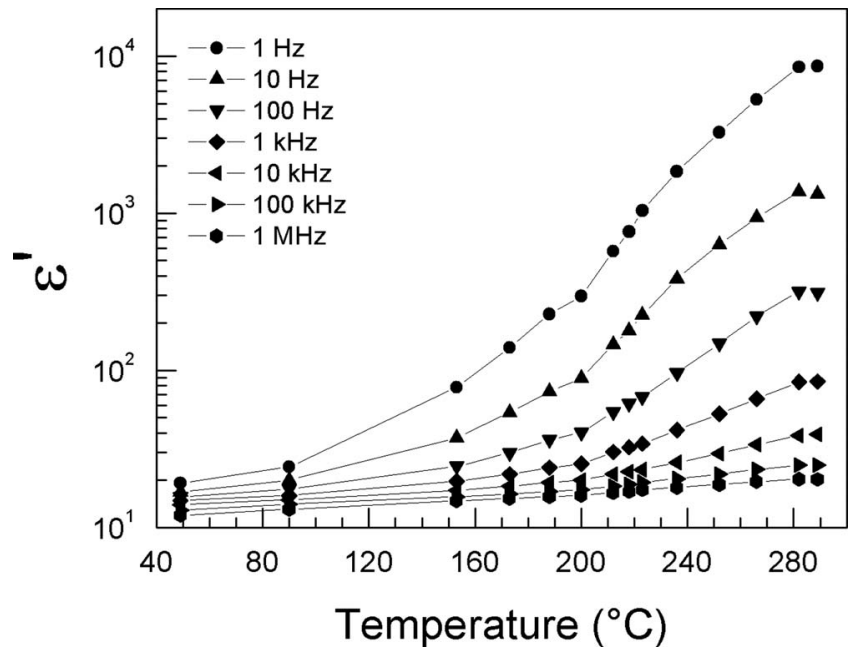

FIG. 5. Temperature dependence of the measured dielectric constant of the $\mathrm{Ba}_{2} \mathrm{BiSbO}_{6}$ between $1 \mathrm{~Hz}$ and $1 \mathrm{MHz}$. 


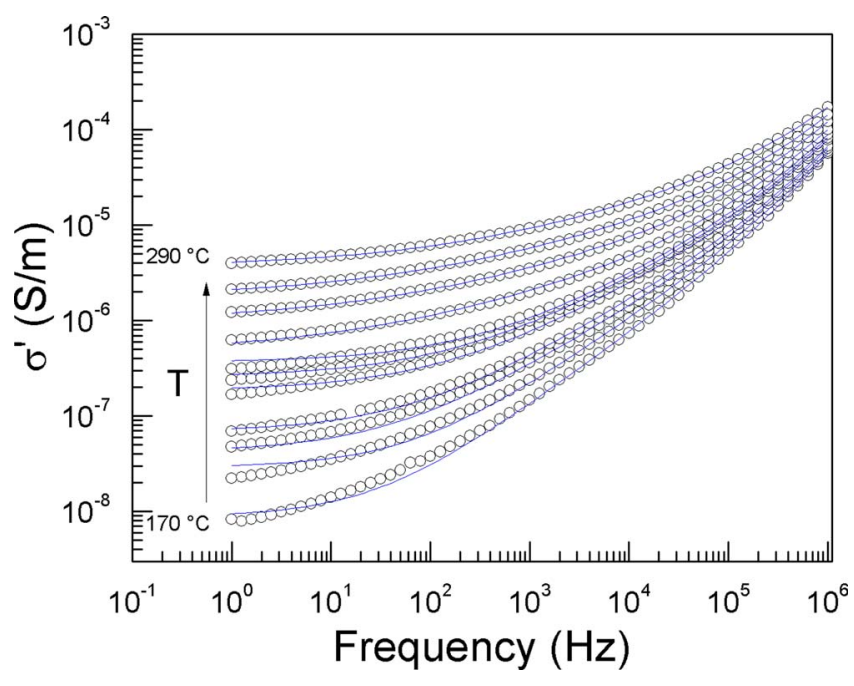

FIG. 6. (Color online) Dependence of the ionic conductivity with the frequency of the $\mathrm{Ba}_{2} \mathrm{BiSbO}_{6}$ at several temperature values. The line indicates the best fit using Eq. (1).

imaginary, $\varepsilon_{o}$ is the vacuum permittivity, and $\varepsilon(\omega)$ is the dielectric constant of the sample obtained from the impedance response through the relation $\varepsilon(\omega)=\left[j \omega C_{o} Z(\omega)\right]^{-1}$, where $C_{o}$ is the capacitance of the open cell. Figure 6 shows the dependence with the frequency of the ac conductivity at several temperatures. Due to grain boundary effects we suppose a frequency dependent conductivity $\sigma(\omega)$ of the form ${ }^{47}$

$$
\sigma(\omega)=\sigma_{0}+A \omega^{s 1(T)}+B \omega^{s 2(T)},
$$

where $A$ and $B$ are temperature dependent constants, $s 1(T)$ and $s 2(T)$ are two nearly temperature independent parameters close to unity, and $\sigma_{0}$ is correlated with the dc conductivity. The best fit of the conductivity at each measured temperature is shown in Fig. 6 as a line. In Fig. 7 we show the temperature dependence of the calculated $s 1(T)$ and $s 2(T)$ parameters. There is an interesting discontinuous change in both parameters that was not observed in the other measured electrical variables. These discontinuous changes reflect the structural phase transitions that the $\mathrm{Ba}_{2} \mathrm{BiSbO}_{6}$ compound undergoes near $242{ }^{\circ} \mathrm{C} .{ }^{30}$ There are discontinuities to the

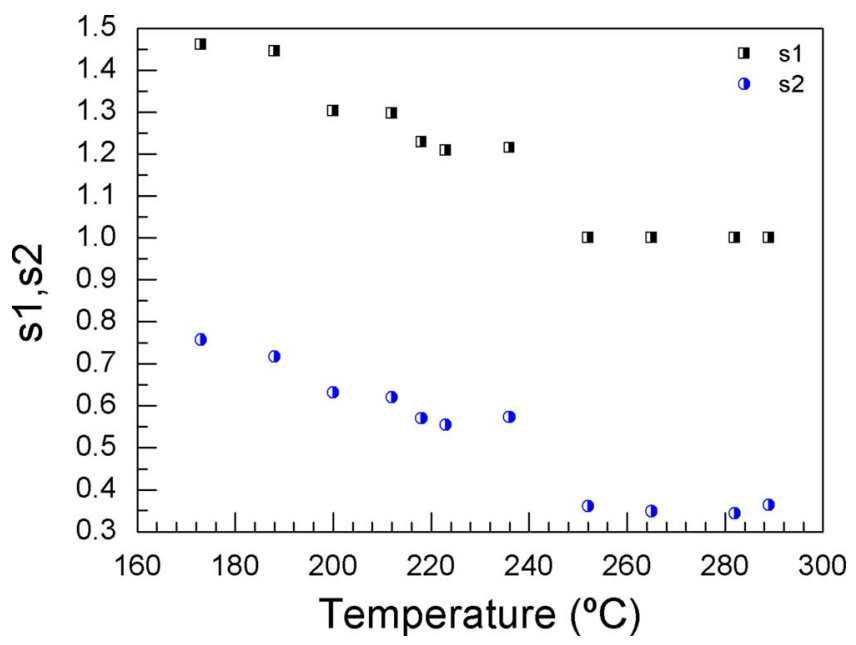

FIG. 7. (Color online) Temperature dependence of the $s 1(T)$ and $s 2(T)$ calculated parameters.

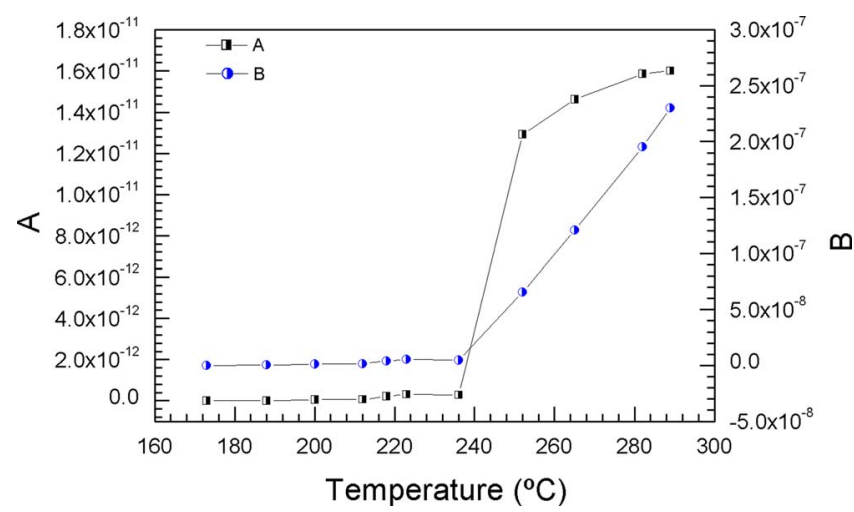

FIG. 8. (Color online) Temperature dependence of the $A$ and $B$ calculated parameters.

same temperature in the $A$ and $B$ estimated parameters as shown in Fig. 8. We can see that the frequency dispersion terms (A and B) influence increases in conductivity considerably above the temperature of the structural phase transition.

\section{CONCLUSION}

In this work we have investigated for the first time the electrical properties of the $\mathrm{Ba}_{2} \mathrm{BiSbO}_{6}$ double complex perovskite using impedance spectroscopy under frequency ( $\mathrm{rf}$ range) and temperature changes. The results indicate that there are two relaxation processes in the ceramic associated to grain and grain boundary. The relaxation due to the grain is due to the oxygen vacancies. These vacancies contribute strongly to the dielectric constant through the ac conductivity. The conductivity was analyzed with basis in a model that accounts for two frequency dependent exponential terms due to the grain and boundary grain conductions. The parameters associated with these contributions clearly show the structural phase transition that $\mathrm{Ba}_{2} \mathrm{BiSbO}_{6}$ undergoes near $242{ }^{\circ} \mathrm{C}$.

\section{ACKNOWLEDGMENTS}

The Brazilian authors acknowledge the financial support from CNPq, FINEP, and FAPEMA Brazilian funding agencies. MWL acknowledges financial support from Research Corporation.

${ }^{1}$ A. S. Bhalla, R. Y. Guo, and R. Roy, Mater. Res. Innovations 4, 3 (2000).

${ }^{2}$ X. F. Long and Z. G. Ye, Appl. Phys. Lett. 90, 112905 (2007).

${ }^{3}$ X. F. Long and Z. G. Ye, IEEE Trans. Ultrason. Ferroelctr. Freq. Control 54, 2595 (2007).

${ }^{4}$ A. A. Bokov and Z. G. Ye, Phys. Rev. B 65, 144112 (2002).

${ }^{5}$ B. Mihailova, U. Bismayer, B. Guttler, M. Gospodinov, and L. Konstantinov, J. Phys.: Condens. Matter 14, 1091 (2002).

${ }^{6}$ A. A. Bokov and Z. G. Ye, Phys. Rev. B 66, 064103 (2002).

${ }^{7}$ M. Tyunina, J. Levoska, K. Kundzinsh, and V. Zauls, Phys. Rev. B 69, 224101 (2004).

${ }^{8}$ M. T. Sebastian and K. P. Surendran, J. Eur. Ceram. Soc. 26, 1791 (2006).

${ }^{9}$ T. Takahashi, Jpn. J. Appl. Phys., Part 1 39, 5637 (2000).

${ }^{10}$ K. P. Surendran and M. T. Sebastian, J. Mater. Res. 20, 2919 (2005).

${ }^{11}$ H. Wu and P. K. Davies, J. Am. Ceram. Soc. 89, 2271 (2006).

${ }^{12}$ S. B. Desu and H. M. Obryan, J. Am. Ceram. Soc. 68, 546 (1985).

${ }^{13}$ D. A. Sagala and S. Nambu, J. Am. Ceram. Soc. 75, 2573 (1992).

${ }^{14}$ A. Dias, C. W. A. Paschoal, and R. L. Moreira, J. Am. Ceram. Soc. 86, 1985 (2003). 
${ }^{15}$ A. Dias, L. A. Khalam, M. T. Sebastian, C. William, C. W. A. Paschoal, and R. L. Moreira, Chem. Mater. 18, 214 (2006).

${ }^{16}$ H. Sreemoolanadhan, R. Ratheesh, M. T. Sebastian, and P. Mohanan, Mater. Lett. 33, 161 (1997).

${ }^{17}$ L. A. Khalam, S. Thomas, and M. T. Sebastian, J. Am. Ceram. Soc. 90, 2476 (2007).

${ }^{18}$ L. A. Khalam and M. T. Sebastian, Int. J. Appl. Ceram. Technol. 3, 364 (2006).

${ }^{19}$ L. Abdul Khalam, H. Sreemoolanathan, R. Ratheesh, P. Mohanan, and M. T. Sebastian, Mater. Sci. Eng., B 107, 264 (2004).

${ }^{20}$ A. W. Sleight, Abstr. Pap.-Am. Chem. Soc. 170, 74 (1975).

${ }^{21}$ A. W. Sleight, J. L. Gillson, and P. E. Bierstedt, Solid State Commun. 17, 27 (1975).

${ }^{22}$ B. Batlogg, Physica B\&C 126, 275 (1984).

${ }^{23}$ T. Nakamura, S. Kose, and T. Sata, J. Phys. Soc. Jpn. 31, 1284 (1962).

${ }^{24}$ V. V. Bogatko and Y. N. Venevtsev, Inorg. Mater. 20, 106 (1984).

${ }^{25}$ V. V. Bogatko and Y. N. Venevtsev, Fiz. Tverd. Tela 25, 1495 (1983).

${ }^{26}$ V. V. Bogatko and Y. N. Venevtsev, Izv. Akad. Nauk SSSR, Fiz. Atmos. Okeana 47, 637 (1983)

${ }^{27}$ C. Chaillout, J. P. Remeika, A. Santoro, and M. Marezio, Solid State Commun. 56, 829 (1985).

${ }^{28}$ R. P. S. M. Lobo and F. Gervais, Solid State Commun. 98, 61 (1996).

${ }^{29}$ R. P. S. M. Lobo and F. Gervais, Phys. Rev. B 52, 13294 (1995).

${ }^{30}$ B. J. Kennedy, C. J. Howard, K. S. Knight, Z. M. Zhang, and Q. D. Zhou, Acta Crystallogr. B 62, 537 (2006).

${ }^{31}$ K. S. Wallwork, B. J. Kennedy, Q. D. Zhou, Y. Lee, and T. Vogt, J. Solid
State Chem. 178, 207 (2005).

${ }^{32}$ H. D. Zhou and J. B. Goodenough, Solid State Sci. 7, 656 (2005).

${ }^{33}$ Q. D. Zhou and B. J. Kennedy, Solid State Commun. 132, 389 (2004).

${ }^{34}$ M. W. Lufaso, R. B. Macquart, Y. J. Lee, T. Vogt, and H. C. zur Loye, J. Solid State Chem. 179, 917 (2006).

${ }^{35}$ E. F. V. Carvalho, E. M. Diniz, and C. W. A. Paschoal, Comput. Mater. Sci. 40, 417 (2007).

${ }^{36}$ Y. Y. Liu, X. M. Chen, X. Q. Liu, and L. Li, Appl. Phys. Lett. 90, 262904 (2007).

${ }^{37}$ Z. Wang, X. M. Chen, L. Ni, Y. Y. Liu, and X. Q. Liu, Appl. Phys. Lett. 90, 102905 (2007).

${ }^{38}$ J. R. Macdonald and E. Barsoukov, Impedance Spectroscopy: Theory, Experiment, and Applications, 2nd ed. (Wiley-Interscience, New Jersey, 2005).

${ }^{39}$ S. Saha and T. P. Sinha, J. Phys.: Condens. Matter 14, 249 (2002).

${ }^{40}$ A. Dutta and T. P. Sinha, J. Phys. Chem. Solids 67, 1484 (2006).

${ }^{41}$ S. Saha and T. P. Sinha, J. Appl. Phys. 99, 014109 (2006).

${ }^{42}$ A. Dutta and T. P. Sinha, Int. J. Mod. Phys. B 21, 2965 (2007).

${ }^{43}$ A. Dutta, T. P. Sinha, and S. Shannigrahi, Phys. Rev. B 76, 155113 (2007).

${ }^{44}$ V. Prakash, A. Dutta, S. N. Choudhary, and T. P. Sinha, Mater. Sci. Eng., B 142, 98 (2007).

${ }^{45}$ V. Prakash, S. N. Choudhary, and T. P. Sinha, Physica B 403, 103 (2008).

${ }^{46}$ K. Aizu, Phys. Rev. B 2, 754 (1970).

${ }^{47}$ A. Huanosta-Tera, R. de Lira-Hueso, O. Perez-Orta, S. A. PalomaresSanchez, S. Ponce-Castaneda, and M. Mirabal-Garcia, Scr. Mater. 42, 603 (2000). 